\title{
Stand und Perspektiven Ausländischer Direktinvestitionen (ADI) in den Wirtschaften Mittelosteuropas
}

Schlüsselworte: Ausländische Direktinvestitionen, internationale und globale Wertschöpfungskette , Regional Cluster, EU-Ost-Erweiterung, Internationale Arbeitsteilung

Keywords: Foreign Direct Investment, International and Global value added chain, Regional Cluster, EU-EasternEnlargement, International division of labour

Słowa kluczowe: BIZ, międzynarodowe łańcuchy produkcji, klastry regionalne, poszerzenie UE i międzynarodowy podział pracy

\section{Einführung}

Die ADI waren und sind ein wesentlicher Bestandteil der Entwicklung in den Wirtschaften der neuen EU-Mitgliedsländer. Dies zeigte sich nicht nur in Tschechien, Ungarn, der Slowakei, sondern vor allem aber in Polen: „Poland's growth has been based primarily on dynamic exports, strong internal demand, productivity improvements, foreign direct investment (FDI), and the inflow of EU funds. This growth was supported by the buoyant demographics of the 1980s baby boom, as well as a stable banking system. Now, however the environment has changed. Some of the fundamentals, including the volume of FDI, annual productivity, and export growth, are slowing, while others, such as the flow of EU funds, will likely narrow after 2020،“1

Auch in Zukunft bleiben die mittelosteuropäischen Wirtschaften wichtige Standorte für den Einkauf, die Fertigung und den Absatz von Produkten aus internationalen

* Prof. Dr. Sc. Harald Zschiedrich; University of Applied Sciences (HTW Berlin), Department: Business Administration, E-Mail: harald.zschiedrich@htw-berlin.de.

1 Vgl. Mc Kinsey \& Germany: Poland 2025; Europe's new growth engine, Jan. 2015, S. 2. 
Unternehmen. (z.B. MTU München in Rzeszow, Mc Kinsey knowledge center in Wroclaw, Shell in Krakow, IBM in Katowice und Wroclaw, etc.)

Wie auch diese Beispiele zeigen, investieren die westlichen Firmen immer mehr in den Dienstleistungssektor. (business service) Schon heute liegt Polen weltweit auf dem dritten Platz beim Offshoring von Business Services; gleich hinter Indien und China: „Almost half of all the FDI in Poland's outsourcing industry has come from banks, insurance and technology companies $-99 \%$ of all outsourced operations serve western European customers (...). Simple tasks may well be going to India, but complex business is coming to CEE, and Poland especially. “2

Somit wird deutlich, dass in den letzten Jahren ein deutlicher Wandel mit Blick auf die Zielsektoren der FDI stattgefunden hat. Genauso wichtig ist jedoch der Umstand, dass die CEE-Locations ihr ausschließliches Image als Billiglohnstandorte verloren haben, denn der sich heute vollziehende Übergang von einer rein nationalen zu einer globalen Wertschöpfungskette erhöht die internationale Wettbewerbsfähigkeit vor allem durch die Aktivitäten der Tochtergesellschaften, durch Offshoring oder durch den Einkauf von Zwischenprodukten in den MOE-Ländern.

\section{Entwicklungstrends von ADI in Mittelosteuropa}

Wie die Wirtschaftsgeschichte klar zeigt, sind ausländischen Direktinvestitionen in Mittelosteuropa kein Phänomen, das wir erst mit Beginn der Transformationsperiode in dieser Region betrachten können. So können wir schon lange vorher Direktinvestitionen in dieser Region registrieren. (vgl. Tabelle 1)

Tabelle 1. FDI in Ostmitteleuropa in der Welt (Bestände in \%) im Zeitraum 1914-2002

\begin{tabular}{|l|c|c|c|c|c|}
\hline & 1914 & 1938 & 1963 & 1990 & 2002 \\
\hline Ostmittel- und Osteuropa & 9,9 & 1,6 & - & $0,1^{\mathrm{a}}$ & $3,1^{\mathrm{a}}$ \\
\hline Westeuropa & 7,8 & 7,4 & 22,9 & 40,8 & 41,6 \\
\hline USA & 10,3 & 7,4 & 13,9 & 20,3 & 20,4 \\
\hline Kanada & 5,7 & 9,4 & 23,7 & 5,8 & 3,0 \\
\hline Japan & 0,2 & 0,4 & 0,2 & 0,5 & 1,0 \\
\hline Australien, Südafrika & 3,2 & 8,0 & 6,6 & $4,9^{\mathrm{b}}$ & $2,8^{\mathrm{b}}$ \\
\hline Entwicklungsländer & 62,8 & 65,7 & 32,3 & 27,6 & 28,0 \\
\hline
\end{tabular}

Quelle: Willkommene Investoren oder nationaler Ausverkauf? - Ausländische Direktinvestitionen in Ostmitteleuropa, Hrsg J. Günther, D. Jajesniak-Quast, Berlin 2006, BWV, S. 39.

\footnotetext{
2 Vgl Cost focused business a path for Poland, „Financial Times“, 23.01.2015, S. 16.
} 
Ein gemeinsamer Wesenszug der Direktinvestitionen bis in die jüngste Vergangenheit hin scheint die Tatsache zu sein, dass sie mitunter als etwas ,vorbelastet" in den Augen der Empfänger in den mittelosteuropäischen Ländern galten, was sich in Ägsten vor ,Fremdbestimmung' und Verdrängung lokaler Firmen vor allem in den Jahren der marktwirtschaftlichen Transformation zeigte. (zum Beispiel in Polen) Gleichzeitig hat sich andererseits auch die Erkenntnis gefestigt, dass die Direktinvestitionen zu einem ganz wichtiger Faktor in der makro- und mikroökonomischen Entwicklung dieser mittelosteuropäischen Staaten geworden sind.

Allerdings bleibt die ökonomische Bewertung der Effekte von Direktinvestitionen bis heute nicht unumstritten (vgl. Zschiedrich, H. 2007; Nummenkamp P. /2005 u. Hunya G. 2010). Unbestritten bleibt der positive Beitrag zu einer höheren Produktivität in den einheimischen Unternehmen, in die ausländische Direktinvestitionen geflossen sind - wie z.B. u.a. Skoda in der CR, Audi in Györ (HU), Daimler (Mercedes) in Kecskemet (HU), Beiersdorf: Polska Nivea in Poznan oder ABB, General Electric, Motorola nach Krakau (Polen) und MTU-München nach Rzeszow (Polen), um nur einige zu nennen. Das Gleiche gilt für die positive Rolle der ausländischen Direktinvestitionen für die Lohnentwicklung, den Ausbau der Exportpotentiale (so entfallen ca. 10\% der gesamten tschechischen Exporte auf diverse Skoda-Modelle) und den Ausbau des Qualifikationsniveaus der Arbeitskräfte in einzelnen Regionen Mittelosteuropas, in die die ausländischen Direktinvestitionen geflossen sind. (Siehe: Aufbau umfangreicher Ausbildungskapazitäten durch Daimler in der ungarischen Region Kecskemet in Vorbereitung des Starts der Fertigung 2012). Nicht so positiv sind die Einflüsse von FDI auf den Abbau regionaler Ungleichgewichte in diesen Ländern gewesen (Zschiedrich, H. 2007), die sich eher in Folge der FDI-Zuflüsse noch vergrößerten. Analoges gilt für die Leistungsbilanzen, die sich im Zuge zunehmender Importe negativ entwickelten. Damit in Verbindung steht die noch nicht voll gelungene Einbindung lokaler / regional gebundener Firmen aus den MOEL in die internationalen Produktionssysteme bzw. Wertschöpfungsketten multi- und transnationaler Unternehmen, wobei ein positiver Trend erkennbar ist, wenn man z.B. die Erfahrungen Ungarns in Betracht zieht: „In den letzten Jahren lässt sich einerseits eine zunehmende Einbettung bestimmter Sektoren in die globalen Produktions- und Handelsketten beobachten, während sich andererseits die Zusammenarbeit mit einheimischen und ausländischen klein- und mittleren Unternehmen, die Zulieferer der großen sind und sich teilweise selbst exportfähig erweisen, verstärkt. Ergänzt wird dieses Bild durch die grenzüberschreitenden Aktivitäten mancher regionalen multinationalen Unternehmen, wie MOL oder OTP in Ungarn, die tschechische CEZ oder ausgewählte polnische und slowakische Firmen.“3

3 Andras Inotai, Auf und ab - Die Wirtschaftslage in den Donauländern, IDM-Reihe / Institut des Donauraumes / Sonderheft 2, 2011, S. 13. 
Ohne wettbewerbsfähige einheimische Zuliefer-Unternehmen können die vielfach in der Literatur diskutierten positiven Wirkungen der FDI kaum zur Entfaltung kommen. Was schließlich die Wirkungen auf die Arbeitsmärkte in MOE betrifft, so können sich ausgehend von den Motiven der konkreten Formen (gemeint sind Greenfield - bzw. Brownfield Investments) unterschiedliche Effekte ergeben: „Obwohl die international operierenden Unternehmen häufig eine klare Präferenz für die Form der Merger \& Acquisition haben, kann der Nutzen dieser Investitionen für die Empfängerländer geringer sein, weil er mit der Verdrängung nationaler Firmen vom nationalen Markt verbunden sein kann. (,Crowding out effect') (...). Markteintritte durch M\&A generierten nicht immer einen Zuwachs an Beschäftigten - im Vergleich zu den positiven Wirkungen der Greenfield Investments, weil damit in der Regel keine neuen Produktionskapazitäten einhergingen." ${ }^{\text {"4 }}$

Insgesamt hat es eine positive Entwicklung der FDI sowohl bereits vor, aber auch nach dem EU-Beitritt 2004 der MOE-Wirtschaften gegeben - wenn auch in unterschiedlichem Maße von Land zu Land. Die Tabelle 2 veranschaulicht deutlich, dass Höchststände in einigen Ländern schon im Vorfeld des EU-Beitritts erzielt werden konnten (Czech Republic, Ungarn). Bei anderen MOE-Wirtschaften kam es zu wesentlichen Steigerungen der FDI-Zuflüsse erst in den Jahren nach dem EU-Beitritt 2004. (z.B. Polen, Slowakei, etc.)

Tabelle 2. FDI-Zuflüsse vor und nach dem EU-Beitritt im Jahr 2004, FDI inflow, EUR Million: 2001-2009

\begin{tabular}{|l|r|r|r|r|r|r|r|r|r|}
\hline & 2001 & 2002 & 2003 & 2004 & 2005 & 2006 & 2007 & 2008 & 2009 \\
\hline Bulgaria & 903 & 980 & 1851 & 2736 & 3152 & 6222 & 9052 & 6697 & 3213 \\
\hline Czech Republic & 6296 & 9012 & 1863 & 4007 & 9374 & 4355 & 7634 & 4415 & 1965 \\
\hline Estonia & 603 & 307 & 822 & 771 & 2307 & 1432 & 1998 & 1317 & 1204 \\
\hline Hungary & 4391 & 3185 & 1888 & 3633 & 6172 & 5609 & 3956 & 4752 & 1021 \\
\hline Latvia & 148 & 269 & 271 & 513 & 568 & 1326 & 1698 & 863 & 52 \\
\hline Lithuania & 499 & 772 & 160 & 623 & 826 & 1448 & 1473 & 1223 & 190 \\
\hline Poland & 6372 & 4371 & 4067 & 10237 & 7112 & 12711 & 15902 & 9601 & 8251 \\
\hline Romania & 1294 & 1212 & 1946 & 5183 & 5213 & 9061 & 7250 & 9496 & 4556 \\
\hline Slovakia & 1768 & 4397 & 1914 & 2441 & 1952 & 3733 & 2382 & 2323 & -36 \\
\hline Slovenia & 412 & 1722 & 271 & 665 & 473 & 513 & 1106 & 1313 & -48 \\
\hline NMS-10 & 22686 & 26227 & 15051 & 30809 & 37148 & 46410 & 52451 & 42001 & 20367 \\
\hline
\end{tabular}

Quelle: Gabor Hunya: FDI in the CEECs Hit Hard by the Global Crisis, in: wiiw-Database on FDI in Central, East and Southeast Europe, Vienna, May 2010, S. 22.

4 Vgl. H. Zschiedrich, Ausländische Direktinvestitionen und Regionale Industriecluster in MOE, 2007, München, S. 61-62. 
In diesem Zeitraum gab es auch erhebliche regionale Umverteilungen der FDI-Zuflüsse zwischen einzelnen Ländern in MOE. So wurden beispielsweise noch 2005 ca. 50\% der neuen Zuflüsse in den 8 neuen Kernländern in MOE registriert, während es 2006 nur noch 36\% waren. Dagegen flossen nach Südosteuropa (Rumänien, Bulgarien, Kroatien) $27 \%$ und in die EU - Staaten 36\%.

Ein Sonderfall in diesem Kontext einer Bestandsaufnahme der FDI-Zuflüsse in die ,Transition economies' sind dabei die Balkanländer. (Albanien, Bosnien-Herzegowina, Bulgarien, Kroatien, Mazedonien, Montenegro, Rumänien und Serbien) Spezielle Untersuchungen haben gezeigt, dass die Länder im Westbalkan wesentlich geringere FDI-Zuflüsse erhielten - im Vgl. zu anderen Transformationsländern. Mit anderen Worten: „Foreign Investors arrived later to most SEE than to CEE and the inflow of FDI to this region in the 1990s was low. The service sector (banking, IT, trade) accounted for the largest part of inward FDI stock in all seven SEE-countries, on average 62\% of the total in the SEE region, but with substantial variations among countries." ${ }^{\text {"5 }}$

Die Transformationsforschung hat insgesamt drei Erklärungen für das relativ niedrige Niveau der FDI-Zuflüsse in die Länder des Westbalkan: ${ }^{6}$

a) Die geringere Größe der Wirtschaften und Märkte im Vgl. zu anderen CEE-Wirtschaften;

b) Die Fragmentierung der Region;

c) Die institutionelle Unterstützung war nicht so stark wie in anderen MOE-Ländern.

Heute haben sich die meisten CEE-Wirtschaften von der weltweiten Finanz- und Wirtschaftskrise weitgehend erholt. (2008-2010) Die Exporte entwickelten sich sogar schneller als vor der Krise, andererseits wurde bei der Industrieproduktion in einigen MOEL das Vorkrisenniveau nicht sofort erreicht. Erschwerend kam hinzu, dass die Märkte in der Eurozone, die zu den größten Absatzmärkten der MOEL gehören, langsamer wuchsen. (2011-2014) Dennoch blieb und bleibt Ost- und Mitteleuropa ein wichtiges Ziel für ausländische Investoren. So hat sich z.B. die Anzahl neuer Greenfieldprojekte nach der Krise (2011/12) deutlich erhöht, wobei damit jedoch weniger FDI verknüpft waren als vorher. Die wichtigsten EU-Herkunftsländer bei FDI blieben und bleiben die Niederlande, Deutschland und Österreich, wobei für deutsche Unternehmen immer mehr China als Zielregion Nr. 1 neben anderen Ländern (Singapur, Indonesien, Vietnam, Türkei, Mexiko, Brasilien) in den Mittelpunkt rückt??

5 S. Estin, M. Uvalic, FDI into transition economies are the Balkans different?, in: Economies of Transition, 2014, vol. 22 (2), S. 281-312.

6 Vgl. ibidem, S. 309.

7 Vgl. Das Kapital kehrt zurück: ADI legen zu, Investoren mit weniger Gewinnen, in: Ost-West-Contact, Berlin 2011, H. 7, S. 17-19. 


\section{Regionale Cluster als Magnet für ADI in Mittelosteuropa}

Die Öffnung der Märkte sowie der damit verbundene Abbau von tarifären und nichttarifären Handelshemmnissen waren in den Jahren der Transformation wichtig, um zunehmende Zuflüsse von FDI in die mittelosteuropäischen Wirtschaften zu generieren.

Heute und in Zukunft wird dies allein nicht mehr ausreichen, um ausländische Direktinvestitionen anzuziehen. Neben einem investitionsfreundlichen, wirtschaftlichen und dem politischen Klima sowie der Entwicklung international wettbewerbsfähiger KMUs erweisen sich regionale Produktion- und Dienstleistungscluster als ein Dreh- und Angelpunkt: „There is a need to develop attractive configurations of locational advantages and value added chains -H.Z.) Competition is no longer between nations, but between strongly networked regions, so-called Clusters." ${ }^{\text {"8 }}$

Welche Eigenschaften/Merkmale sind es im Einzelnen, die regionale Produktionsbzw. Dienstleistungscluster heute so begehrt machen für die Unternehmen im Allgemeinen und die ausländischen Investoren im Besonderen?

Cluster werden meist als ein regionales Konzept begriffen. Es handelt sich hierbei um eine Ballung komplementärer Industriebranchen und unterstützender Institutionen, die in einer Wertschöpfungskette miteinander Synergieeffekte erzeugen - durch vielfältige Absatz-, Arbeitsmarkt- und Lernbeziehungen.

Clusterkonzepte sind besonders für kleine und mittlere Unternehmen interessant, da sie aufgrund begrenzter eigener Ressourcen von diesen Synergieeffekten besonders profitieren und ihre Innovationskraft stärken können. Sie erhalten nicht nur Anregungen zur Entwicklung neuer Produkte und Technologien, sondern darüber hinaus auch Gelegenheiten, internationale Marktchancen zu ergreifen und Partnerschaften aufzubauen.

Wichtiger ist also eine starke räumliche Konzentration branchenverwandter Industrie- und Dienstleistungsunternehmen: „Clusters are a concentration of firms in one of our few industries, benefiting from synergies created by a dense network of competitors, buyers and suppliers. Clusters comprise demanding buyers, specialized suppliers, sophisticated human resources, finance and well-developed support institutions. “9

Von zentraler Bedeutung hat sich dabei auch für die Clusterbildung in MOE der von M. Porter entwickelte Ansatz erwiesen: „Cluster sind eine Organisationsform von Industrien, die höhere Produktivität und mehr Innovationen erzeugen als räumlich weiter aufgefächerte Strukturen. In einem Cluster stehen sich auf relativ begrenztem Raum verschiedene Unternehmen und Institutionen gegenüber, die alle auf den Wettbewerb

\footnotetext{
8 J. Kluge (Mc Kinsey), Cluster on the rise as European's border fall, in: Prop Europe, 2003, Düsseldorf, S. 75 .

9 Vgl. World Investment Report, Genf 2002, S. XIX.
} 
einwirken: Hersteller, Zulieferer, Dienstleistungsanbieter, Universitäten und andere Ausbildungsstätten (z.B. Fachhochschulen)."10

Für den Bezug zu den ausländischen Direktinvestitionen ist vor allem der Zusammenhang mit den international verflochtenen Wertschöpfungsketten der Unternehmen wichtig. Nach M. Porter wird danach unter einer Wertschöpfungskette ein branchenübergreifender Zusammenhang von Unternehmen verstanden, die an der Herstellung eines bestimmten Produktes oder einer Dienstleistung arbeitsteilig beteiligt sind. Dabei ist es heute üblich, dass - auch unter Einbeziehung mittelosteuropäischer Firmen - die Wertschöpfungskette mit ihren einzelnen Gliedern über eine Vielzahl von Unternehmen an unterschiedlichen Standorten in unterschiedlichen Ländern/Regionen verteilt ist, wobei zwischen ,Clusterkern' (Kernkompetenzen) und ,Clusterumfeld' (Zulieferfirmen, Institutionen, etc.) zu unterscheiden ist. Somit liegt heute ein regionales Cluster vor, wenn die Funktionen einer Wertschöpfungskette über eine Vielzahl von Unternehmen und anderer Organisationen verteilt und zahlreich innerhalb einer bestimmten Region vertreten sind, sowie die Akteure intensive Austausch- und Kommunikationsbeziehungen unterhalten.

Die bisherigen Erfahrungen in Mittelosteuropa bei Automobilclustern (Tschechien; Mlada Boleslav; Györ/Kecskemet in Ungarn; Peugot in Kolin; VW/Porsche-Bratislava oder Renault: Pitesti in Rumänien) haben eindrucksvoll bestätigt, dass die in Cluster eingebundenen Firmen aus MOE größere Erfolgsaussichten haben und international wettbewerbsfähiger sind als andere einheimische Firmen. (vgl. Fallbeispiel 1)

\section{Fallbeispiel 1:}

Das mittelosteuropäische Automobilcluster - Ziel von FDI.:

a) Charakteristisch ist, dass im Zentrum der einzelnen regionalen Automobilcluster in MOE jeweils ein westliches Großunternehmen steht, dessen Investitionen und Technologie - sowie Human Ressources-Transfers die Grundlage der Clusterbildung sind: Mlada Boleslav) Volkswagen; Trnava (SK) - Volkswagen, Bratislava - VW/Porsche; Hyundai / KIA: Zilina (SK); Suzuki: Esztergom (HU); Opel: Gliwice (Polen); Audi: Györ (HU), Mercedes (Daimler): Kecskemet (HU) und Renault (Dacia) in Pitesti (RU), wobei Renault 2013 mit 290000 die meisten Neuwagen ( $23 \%$ mehr als im Vorjahr) verkaufte!

b) Hinzukommen lokale Produktionen internationaler Marken in Russland wie z.B. BMW, Ford, Hyundai, KiA, Volkswagen (Kaluga), GM und Renault, in deren Ergebnis 4 russische PKW-Automobilcluster entstanden;

10 M. Porter, Erfahrungen aus Clusterstudien in den USA, in: Cluster-McK-Wissen, Mc Kinsey Company, Hamburg 2002, S. 21. 
c) Jeder fünfte im Ausland gefertigte PKW (2013) mit deutschem Markenzeichen hatte seinen Standort in MOE. Die Slowakei hat die höchste Pro-Kopf PKWProduktion weltweit!

d) Insgesamt produzierte die MOE Region 2013 1,5 Mio. PKW.

In der Zukunft werden solche übergreifenden Firmenkooperationen zwischen den MOE-Unternehmen und den multinationalen Unternehmen immer wichtiger. Dies gilt auch für die Verflechtungen zwischen Wirtschaft und Wissenschaft in MOE, wie man das am Beispiel des 'knowledge-based Cluster' in Krakau (Polen) beobachten kann:

\section{Fallbeispiel 2:}

Das knowledge-based Cluster in Krakow:

a) Aufbau eines Kompetenzzentrums des deutschen Großanlagenbauers Lurgi für Methanol-, Wasserstoff-, und Bio-Ethanolanlagen;

b) Krakau auf dem Weg zu einem FuE-Mekka internationaler Großunternehmen, zu einer Denkfabrik für komplexe Konstruktions- und Entwicklungsleistungen;

c) 10 multinationale Unternehmen haben eigene FuE-Zentren, wie z.B.: Motorola, IBM, ABB, Delphi und Google (Programmierer von Suchmaschinen);

d) Typisch für dieses wissensbasierte Cluster Krakow ist das enge Zusammenwirken von Universitäten, technischen Instituten, Großunternehmen und Zulieferunternehmen (ca. 55 Tausend Studenten erwerben pro Jahr einen technischen/ naturwissenschaftlichen Abschluss);

e) Besseres Angebot an FuE-Ingenieuren als in Deutschland;

f) $\mathrm{ABB}$ verlegt die Konzernaufsicht über die Einhaltung internationaler Industrienormen von Schweden / Schweiz ins polnische Krakow;

g) Fazit: Es ist nur eine Frage der Zeit, bis die Innovationskraft eines solchen FuEClusters wie in Krakow auf die gesamte polnische Wirtschaft übergreift (Spillover Effect).

Clusterbildungen sind international gesehen auch ein Mittel für die Herausbildung ,stabiler Standorte in MOE und begünstigen die Standorttreue von Beschäftigten und Firmen, was auch japanische Forschungen eindeutig belegen, wenn man berücksichtigt, dass sich in Zukunft die MOE-Firmen immer stärker von einer ,verlängerten Werkbank zu einer ,High-Value Production“ hin bewegen können und müssen: „High Value Production in Japan depends not only on a good quality workforce, but also on a strong local network of specialist sale-contractors to provide parts and services. (Clustered around Osaka and Kyoto in central Japan). If our clients have a problem (with the design of a new part) it is helpful that they have such a short distance to come to talk to us about it (...). 
Such close cooperation is one of the reasons that companies maintain a relatively high number of employees in Japan." ${ }^{\text {"11 }}$

Mögen diese japanischen Erfahrungen auch zu einer Orientierung beim künftigen Aufbau neuer Cluster in den MOE-Wirtschaften werden. Der gezielte Aufbau neuer Cluster wird nicht nur den Zustrom von FDI beschleunigen, sondern auch zu weiteren Effekten führen, wie z.B.:

a) Kommunikationswege werden kürzer und die Marktteilnehmer können rasch aufeinander reagieren;

b) In Clusterräumen (z.B. Poznan) werden Güter erzeugt, auf die dort angesiedelte Firmen relativ kostengünstig zurückgreifen können;

c) Clusterbildungen fördern die Mobilität der Arbeitskräfte in den MOEL (Wechsel von einem Unternehmen zum anderen, z.B. Szekesfehervar in Ungarn);

d) Es folgt ein schneller Zugriff auf Kapital, wobei Finanzinstitutionen, die in einem Cluster aktiv sind, ihre branchenspezifischen Erfahrungen nutzen (z.B. Volkswagen Bank - Autoindustrie);

e) Die Wege von der Grundlagenforschung in MOE zur anwendungsorientierten Planung und zur Serienfertigung entlang einer international verflochtenen Wertschöpfungskette werden einfacher und schneller (vgl. Fallbeispiel).

\section{Wie geht es weiter?}

Im Unterschied zu russischen Firmen ist es doch ausgewählten Unternehmen aus Polen, Slowakei, Tschechien, Ungarn, Rumänien und Bulgarien bereits gelungen, sich in internationale Wertschöpfungsketten einzubinden. Darauf lässt sich - wie obige Darlegungen zeigten - durchaus aufbauen. Internationale Produktionssysteme - dies belegten Erfahrungen transnationaler Unternehmen wie Siemens, ABB oder Samsung kennen heute keine nationalen Grenzen mehr. Dies zeigt sich auch darin, dass immer mehr deutsche Unternehmen bei ihren Exportprodukten für den Weltmarkt gern auf Vorleistungsimporte aus MOE-Firmen, in die sie vorher investiert haben, zurückgreifen. Bereits heute ,stecken' im gesamten Export der deutschen Industrie 42\% Vorleistungsimporte - Tendenz steigend! Westliche Unternehmen fühlen sich bereits jetzt und in naher Zukunft motiviert, in MOE-Unternehmen zu investieren, wenn sich diese als ein zuverlässiges und starkes Glied einer internationalen Wertschöpfungskette erweisen. Dabei ist davon auszugehen, dass neue technische Entwicklungen in der Zukunft (Industrie 4.0) zu einer zunehmenden Fragmentierung der Wertschöpfungsketten führt. (Called by Krugman ,slicing up the value added chain').

11 P. Marsh, Masters of the craft: How Japanese industrie beat off low-cost competition, „Financial Times“, 5, 2005, S. II. 
Somit vertiefen die globalen Wertschöpfungsketten künftig immer mehr die internationale Arbeitsteilung, indem sie gezielt die Investitionsströme an die Standorte lenken, wo die einzelnen Segmente der globalen Wertschöpfungskette angesiedelt werden sollen.

Mit anderen Worten: Europäische und asiatische Unternehmen werden diese Entwicklungen aufgreifen, um in ausgewählten regionalen Clusterräumen in MOE in einzelne Segmente der Wertschöpfungskette zu investieren - nach dem Motto: „From high volume to high value“ (R. Reich). Die ausländischen Direktinvestitionen werden dabei helfen, die einzelnen Segmente Fertigung, Forschung, Entwicklung, Beschaffung und Vertrieb dort zu etablieren, wo sie am effizientesten - darunter auch in MOE - durchgeführt werden können. Somit können wir davon ausgehen, dass bei künftigen Investitionsaktivitäten in MOE nicht mehr vorrangig die Nutzung niedriger Lohnkosten im Zentrum stehen wird, sondern eher die Optimierung der Wertschöpfungskette und die Nutzung der Absatzmärkte. Das ist eine Chance und Herausforderung für die Unternehmen in MOE, denn es geht darum, neue regionale und lokale Wertschöpfungsketten aufzubauen, um mit eigenen wettbewerbsfähigen Industrieerzeugnissen oder Dienstleistungen (vgl. ABB in Krakau oder MTU in Rzeszow) näher am MOE-Kunden zu sein.

Gesamtwirtschaftlich bzw. makroökonomisch steht im Vordergrund, die Wirtschaftsregion in MOE nach der Krise 2008-2011 wieder auf einen Wachstumspfad zurückzuführen. (bereits 2012 erreichte die Region als Ganzes ein Wirtschaftswachstum von 3,8\%!) Neben den inneren Kernfragen wie die Verbesserung der Exportpotenziale, die Stärkung der inneren Nachfrageentwicklung, die Zunahme privater Investitionen wird auch das internationale weltwirtschaftliche Umfeld einen großen Einfluss auf die potenziellen FDI-Zuflüsse nach MOE haben. Dies bedeutet, dass andere Investitionszielstandorte in Mexiko, Türkei, Vietnam, Singapur, Indonesien und natürlich Indien, China sowie mittelfristig auch Russland noch stärker in den Mittelpunkt ausländischer Investoren treten werden. In diesem Sinne wird die MOE-Region, die überragende Rolle, die sie für westliche EU-Investoren bis ca. 2008 inne hatte, verlieren. Soll an die früheren Erfolge bei FDI-Zuflüssen angeknüpft werden, so rücken folgende Aspekte in den Fokus von Investitionsentscheidungen in MOE, wie z.B.:

- Die verbesserte internationale Wettbewerbsfähigkeit der Unternehmen in den MOE-Zielländern,

- Die Schaffung eines attraktiven Investitionsklimas,

- Die politische Stabilität (vgl. aktuelle Entwicklungen in Ungarn),

- Die effizientere Arbeit der Investitionsagenturen sowie anderer Ämter und Behörden.

Wenn auch der große, Investitionsboom ' der 90er Jahre vorbei zu sein scheint, so haben doch die MOE-Länder auch künftig - neben den immer noch relativ niedrigen 
Arbeitskosten (1:5) - auch noch andere komparative Vorteile in die Waagschale zu werfen, die künftig für potenzielle Investoren von Interesse sind:

a) Der Aufschwung bei Unternehmensgründungen und die Herausbildung unternehmerischer Talente in dieser MOE-Region (z.B. Steve Wozniak als Entwickler des Apple Computers);

b) Produktionen von mehr, value-added goods and services';

c) Weg vom, low-cost image' oder der, verlängerten Werkbank' hin zu ,High-ValueErzeugnissen;

d) Gute geographische Lage und Zugang zu den GUS-Märkten sowie zum Westbalkan;

e) Exzellenter logistischer Service und Infrastruktur (z.B. in Ungarn und Polen);

f) Flexible Arbeitsmärkte mit komparativ noch niedrigeren Arbeitskosten (1:5);

g) Gewährung von Investitionsförderungen durch die nationalen Regierungen;

h) Die Automobilindustrie als Objekt von FDI reicht allein nicht mehr aus. Ins Zentrum von FDI werden zunehmend auch die Pharmazie, die IT-Industrie, Elektronik, Medizintechnik, Lebensmittelindustrie und Chemie treten.

\section{Schlussbemerkungen}

Der Standort Mittelosteuropa wurde von der großen internationalen Finanzkrise sehr stark getroffen. Die Krise zeigte gleichzeitig eine Reihe von ,hausgemachten' Schwächen auf wie z.B. zu geringe Mittel in Forschung und Entwicklung, nicht überall befriedigendes Business - bzw. Investitionsklima, schwache Innovationsbereitschaft, nicht ausreichende Produktivität in vielen KMUs, die Lücke zwischen den TNC / MNU und den KMUs, Defizite in der praxisorientierten Hochschulausbildung sowie eine Erweiterung der regionalen Ungleichgewichte.

Die Differenzierung der Investitionsstandorte wird innerhalb von MOE voranschreiten. Neben weiterhin erfolgreichen Standorten vor allem in Polen, in Tschechien, Rumänien oder der Slowakei wird es weiterhin Standorte geben, wo politische und wirtschaftliche Rahmenbedingungen das Investitionsumfeld erschweren (Ungarn, Russland).

Hinzu kommt, dass künftig MOE nicht mehr vor allem als Absatzmarkt, sondern stärker als Produktions- und Beschaffungsmarkt genutzt werden wird. Das Ganze wird sich vollziehen vor dem Hintergrund eines generell stärkeren Ringens um ausländische Investoren: „CEE-Countries will have to compete hard for slices of a smaller investment pie. Investment will go in the future where the investment climate is the best." ${ }^{\text {"12 }}$

12 N. Buckley, Competition is a fierce for slices of a smaller pie, ,Financial Times“, 5.06.2013, S. 3. 


\section{Literaturverzeichnis}

Heribert D.,.Deutschland in der Weltwirtschaft: Ein Modell mit Zukunft?, Bonn 2013.

Hagemejer J., Tyrowicz J., Is the effect really so large? Firm-level evidence on the role of FDI in a transition economy, „Economics of Transition“, 2012, vol. 20 (2).

Hunya G., FDI in the CEEC. Hit hard by the global crisis, The Vienna Institute for International Economic Studies (wiiw), database on FDI in CEE, Vienna 2010.

Huwart J., Verdier L., Die Globalisierung der Wirtschaft- Ursprünge und Auswirkungen, OECD (Hrsg.), Paris 2014.

Kralemann M., Schröder A., Sonnabend M., Treibhäuser der Innovation - Clusterpotenziale für Wirtschaft und Wissenschaft, Stifterverband für die Deutsche Wissenschaft, Essen 2007.

Love P., Lattimore R., Internationaler Handel: Frei, fair und offen, OECD (Hrsg), Paris 2009.

Nagy B., Analyse möglicher Wirkungen der Merce der Direktinvestition auf die Beschäftigtenentwicklung der Region Kecskemet (Ungarn), Göttingen 2012 (Masterthesis).

Nagy B., Zschiedrich H., How FDI affects a sub region in Hungary - a case study of Daimler in Kecskemet, Präsentation auf der East-West Wirtschaftskonferenz der WU, Wien 2012.

Scherer R., Brieger T., Clustering: Clusterung - das Zauberwort der Wirtschaftsförderung, Verlag Paul Haupt, Bern 2004.

Wilkommene Investoren oder nationaler Ausverkauf? - Ausländische Direktinvestitionen in Ostmitteleuropa, hrsg. J. Günther, D. Jajesniak-Quast, Berlin 2006, BMV.

Zschiedrich H., Ausländische Direktinvestitionen und Regionale Industriecluster in MOE, Rainer Hampp Verlag, München 2007.

Zschiedrich H., Christians U., Grenzüberschreitende Kooperationen - Erfahrungen deutscher und polnischer Mittelständischer Unternehmen und Banken, Rainer Hampp Verlag, München 2009.

Zschiedrich H., Guyda A., Industrial Clusters, FDI and regional Disparities in CEE, Vienna 2011, Presentation at the WU December 2011.

Zschiedrich H., Hummel W., Cross-border cooperation between Polish and German SMEs: Preconditions, barriers and experience in the Berlin-Brandenburg region, Vienna 2010, Conference Proceedings of the WU.

Zschiedrich H., (Hrsg), Deutsch-polnische grenzüberschreitende Unternehmenskooperation Erfahrungen und Wirkungen, Berlin 2012 (BWV).

\section{Abstract}

Vor zehn Jahren waren im Kontext der EU-Osterweiterung teilweise die Ängste größer als die Hoffnungen, die sich mit einer Erweiterung der EU verbanden - war es doch die größte EU-Erweiterung, die 74 Millionen Menschen in die Union brachte. Es haben sich in diesen 10 Jahren nicht nur die Ex-und Importe fast verdoppelt. Auch die ausländischen Direktinvestitionen haben eine maßgebliche Rolle für die wirtschaftliche Entwicklung gespielt. Der Beitrag zeigt die Ausgangsbedingungen, die Tendenzen und den Wandel der Motive für die FDI (Foreign Direct Investment) in den letzten 20 Jahren. Es wird sichtbar, dass sich immer mehr - insbesondere in der Automobilindustrie - regionale Cluster als Anziehungspunkte ausländischer Direktinvestitionen erwiesen haben. Während es vor der Aufnahme der zehn neuen Beitrittsländer aus Mittelosteuropa in die EU vor allem und z.T. ausschließlich die Lohnkostenkostenvorteile waren, die westliche Unternehmen zu Investitionen und Verlagerungen motivierten; geht es jetzt und in Zukunft vor allem darum, regionale Wertschöpfungsketten (unter Einbindung lokaler und regionaler Zulieferer) aufzubauen, die in die internationalen arbeitsteiligen Strukturen der multi- und transnationalen Unternehmen eingebettet sind, um näher 
am Kunden in den internationalen Märkten zu sein. Somit wird Mittelosteuropa ein wichtiger Investitionsstandort auch in Zukunft bleiben, wenn er auch die überragende Rolle, die er für westliche Firmen aus der EU im Zeitraum 1990-2005 hatte, verlieren wird.

Translated by Dr. Lilliane Jodkowski, Berlin/Stettin

\section{State and perspectives of Foreign Direct Investments (FDI) in Central and Eastern Europe}

Ten years ago, in the Western countries, the fears of EU Eastern Enlargement were greater than the expectations. Meanwhile, the mutual exports and imports have doubled since 2004. Also, the FDI-inflows have made a positive contribution to the economic development of the CEE economies, and, above all, to the outstanding growth rates in Poland.

This article is aimed to demonstrate not only the changes in the conditions and the motivations of FDI, but also the new trends concerning FDI development. For example, Regional Industrial Clusters have proved to be important for attracting FDI flows. Step by step, the priorities of the western companies have changed. Today, western firms like more and more to integrate CEE firms into global value added chains. Thus, the CEE firms are more closely involved in the international division of labor structures.

All in all, the CEE economies should remain an interesting place for FDI. However, they will not succeed in keeping the 'number one' position in regard to the FDI activities of the European firms as it happened in the first ten years after the beginning of the transition period.

\section{Stan i perspektywy bezpośrednich inwestycji zagranicznych w Europie Środkowo-Wschodniej}

Przed ponad dziesięciu laty obawy dotyczące poszerzenia UE były większe niż nadzieje, które się z nim wiązałybyło to w końcu największe poszerzenie UE, po którym przybyło Unii $74 \mathrm{mln}$ nowych obywateli. Bezpośrednie inwestycje zagraniczne (BIZ) odegrały duże znaczenie w rozwoju gospodarczym krajów Europy Środkowo-Wschodniej (EŚW). W artykule opisano warunki wyjściowe, tendencje oraz zmiany w motywach dotyczących $\mathrm{BIZ}$ w ostatnich 20 latach. Widoczne przy tym jest, że coraz większą rolę, zwłaszcza w przemyśle samochodowym, odgrywają regionalne klastry, które przyciagają BIZ. Podczas gdy przed przyjęciem do UE 10 nowych krajów członkowskich z EŚW przede wszystkim, a częściowo wyłącznie niższe koszty pracy motywowały zachodnie przedsiębiorstwa do inwestowania i przeniesienia produkcji, w chwili obecnej i na przyszłość chodzi przede wszystkim o stworzenie regionalnych łańcuchów produkcji (przy zaangażowaniu lokalnych i regionalnych dostawców) i włączenie ich do międzynarodowych struktur produkcji wielonarodowych oraz transnarodowych przedsiębiorstw i niewielką odległość od klientów na rynkach międzynarodowych. Tak więc EŚW pozostanie ważnym miejscem inwestycji także w przyszłości, pomimo utraty tej szczególnej roli, którą odgrywała dla zachodnich firm z UE w latach 1990-2005. 